\title{
HIGH ASPECT RATIO TRANSMISSION LINES MICROMACHINED IN SILICON WITH LARGE THERMAL OXIDE DIELECTRICS
}

\author{
S. T. Todd ${ }^{1}$, J. E. Bowers ${ }^{1}$, and N. C. MacDonald ${ }^{2}$ \\ ${ }^{1}$ Department of Electrical and Computer Engineering, University of California, Santa Barbara, CA, USA \\ ${ }^{2}$ Department of Mechanical Engineering, University of California, Santa Barbara, CA, USA
}

\begin{abstract}
A micromachining process has been developed to create high aspect ratio transmission lines on silicon. The transmission lines contain large $\mathrm{SiO}_{2}$ dielectrics that separate the signal and ground conductors. The large $\mathrm{SiO}_{2}$ dielectric is fabricated by merging silicon mesa arrays into solid $\mathrm{SiO}_{2}$ mesas using thermal oxidation. We report measurements of a $40 \Omega$ semi-rectangular coaxial (SERC) line with an effective dielectric constant of 3.7 and attenuation of $1.3 \mathrm{~dB} / \mathrm{cm}$ at $30 \mathrm{GHz}$.
\end{abstract}

\section{INTRODUCTION}

A problem with silicon microwave circuits is that the substrate conductivity causes substantial transmission line loss. Micromachining methods can reduce the substrate loss of silicon. One micromachining approach is to reduce field exposure to the substrate through silicon removal [1]. Micromachining can also be used to lower conductor loss $[2,3]$. Problems with these types of micromachined transmission lines include difficult and expensive fabrication methods and non-planar surfaces.

Previously, high aspect ratio coplanar waveguide (HARC) was created with a planar surface on silicon [4]. A problem with this method was that long thermal oxidation times limited the width of the $\mathrm{SiO}_{2}$ dielectric. In this paper, we introduce high aspect ratio transmission lines with large $\mathrm{SiO}_{2}$ dielectrics. We have created both HARC and SERC with this method and will summarize the results of SERC transmission lines. A mesa merging thermal oxidation process similar to that reported in [5] is utilized to create large dielectrics. The SERC lines have a ground plane that completely isolates the transmission line from the silicon substrate. Fig. 1 shows a 3-D schematic of SERC.

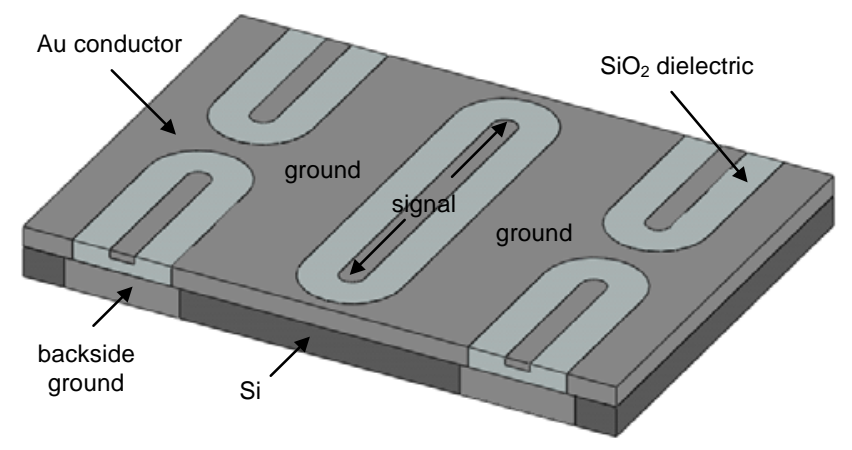

Fig. 1. 3-D schematic of SERC fabricated on silicon.

\section{FABRICATION}

The fabrication method of SERC, shown in Fig. 2, starts with silicon DRIE to create mesas which define the dielectric topology. Next, wet thermal oxidation at $1050{ }^{\circ} \mathrm{C}$ transforms the silicon mesas into oxide, creating a low-loss dielectric for the transmission lines. During thermal oxidation, the width of each mesa expands until it merges with an adjacent mesa. Annealing is then performed at $1100{ }^{\circ} \mathrm{C}$ for 12 hours to ensure mesa merging. Fig. 3 shows SEM micrographs of cross-sections before and after mesa merging.

The surface is then planarized using CMP. Next, a deep anisotropic $\mathrm{SiO}_{2}$ etch forms trenches within the dielectrics that define the center conductor topology. Silicon DRIE follows to define the ground plane trenches. A conformal Ti/Au seed layer is then sputtered. Next, Au electroplating fills both the ground and signal trenches with conductor. The top of the structure is then planarized using lapping and CMP. A backside DRIE follows which removes the silicon underneath the SERC line. Finally, a backside $\mathrm{Ti} / \mathrm{Au}$ seed layer is sputtered followed by backside electroplating. Fig. 4 shows SEM micrographs of the SERC surface.

Before thermal oxidation, the width of each Si mesa is about $4.7 \mu \mathrm{m}$ and the trench width between mesas is about $5.3 \mu \mathrm{m}$ as shown in Fig. 3 (a). Obtaining the correct mesa to trench pitch is important because making the pitch too small results in gaps between the mesas and making the pitch too large results in residual silicon in the center of each mesa. With this mesa to trench width pitch, the mesas contact and merge with no residual silicon left in the center of each mesa. Fig. 3 (b) shows the $\mathrm{SiO}_{2}$ mesa after thermal oxidation and anneal. Notice that a very large $\mathrm{SiO}_{2}$ mesa is created without gaps or residual silicon.

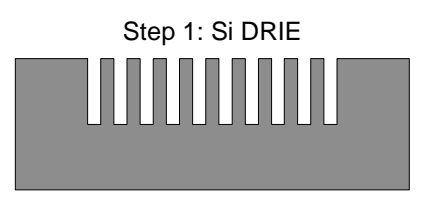

Step 2: Thermal oxidation

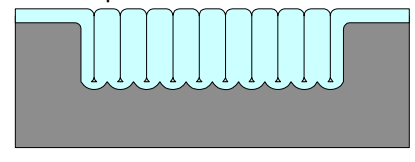

Step 3: Anneal

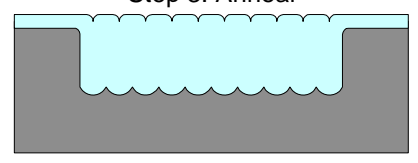

Step 4: Topside CMP

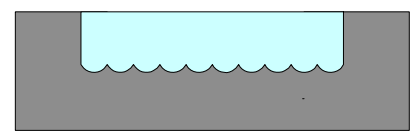

Step 5: Anisotropic $\mathrm{SiO}_{2}$ Etch

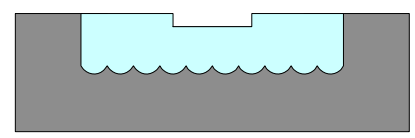

Step 6: Si DRIE

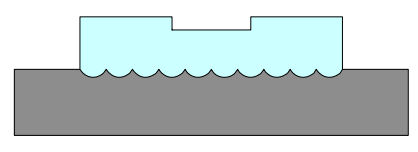

$\square \mathrm{Si} \quad \square \mathrm{SiO}_{2} \quad \square \mathrm{Au}$
Step 7: Ti/Au Seed Layer

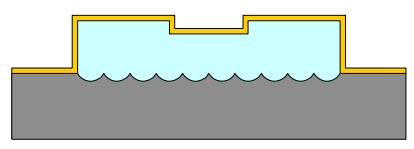

Step 8: Au Electroplating

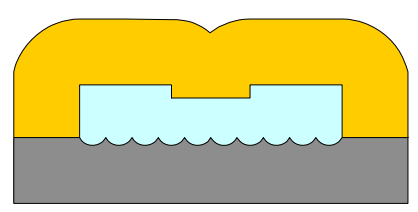

Step 9: Planarization

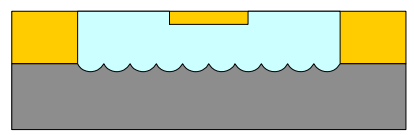

Step 10: Backside Si DRIE

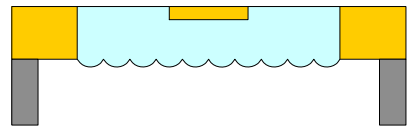

Step 11: Backside Ti/Au Seed Layer

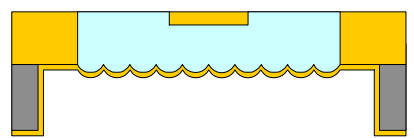

Step 12: Backside Au Electroplating

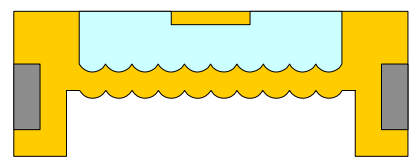

Fig. 2. The process flow for creating SERC. 


\section{EXPERIMENTAL RESULTS}

The SERC transmission line characteristics were extracted from S-parameters that were measured after performing shortopen-load-thru and thru-reflect-line calibrations. Measurements were compared to analytical models that have been derived for SERC. Fig. 5 shows results of characteristic impedance versus frequency of a $400 \mu \mathrm{m}$ long line. Fig. 6 shows results of effective dielectric constant versus frequency of a $400 \mu \mathrm{m}$ long line.

Fig. 7 shows measurements and calculations of attenuation constant versus frequency of a $400 \mu \mathrm{m}$ long line. Notice that the measured loss is higher than the model predicts. We assumed a loss tangent of zero in the model, so the higher than predicted loss could be due to a finite loss tangent. Fig. 8 shows measurements of the loss due to turns in the serpentine SERC line shown in Fig. 4 (b). Fig. 8 shows that the additional loss is less than $0.0006 \mathrm{~dB}$ per $180^{\circ}$ turn over a span of $25 \mathrm{GHz}$. This verifies that turns can be implemented in SERC lines without significant loss.

\section{REFERENCES}

[1] C. Y. Chi and G. M. Rebeiz, "Planar microwave and millimeter-wavelumped elements and coupled-line filters using micromachining techniques," IEEE Trans. Microwave Theory Tech., 43, 4, pp. 730-738, (1995).

[2] J. R. Reid, E. D. Marsh, and R. T. Webster, "Micromachined rectangular coaxial transmission lines," IEEE Trans. Microwave Theory Tech., 54, 8, pp. 3433-3442 (2006).

[3] T. L. Willke and S. S. Gearhart, "LIGA micromachined planar transmission lines and filters," IEEE Trans. Microwave Theory Tech., 45, 10, pp. 1681-1688 (1997).

[4] S. T. Todd, X. T. Huang, J. E. Bowers, and N. C. MacDonald, "High aspect ratio CPW fabricated using silicon bulk micromachining with substrate removal," In Proc. Asia Pacific Microwave Conference 2009, Singapore.

[5] C. Zhang and K. Najafi, "Fabrication of thick silicon dioxide layers for thermal isolation," Journal of Micromech. Microeng., 14, pp. 769-774, (2004).

\section{CONTACT}

S. T. Todd, tel: +1-805-893-5341; stodd@ece.ucsb.edu

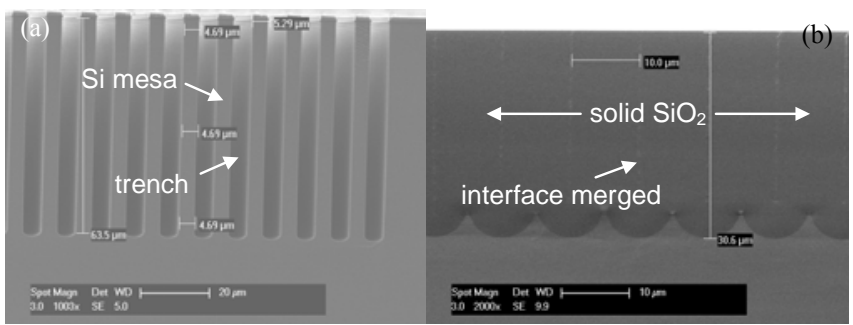

Fig. 3. SEM micrographs of mesa cross sections (a) before thermal oxidation and (b) after oxidation, anneal, and planarization.

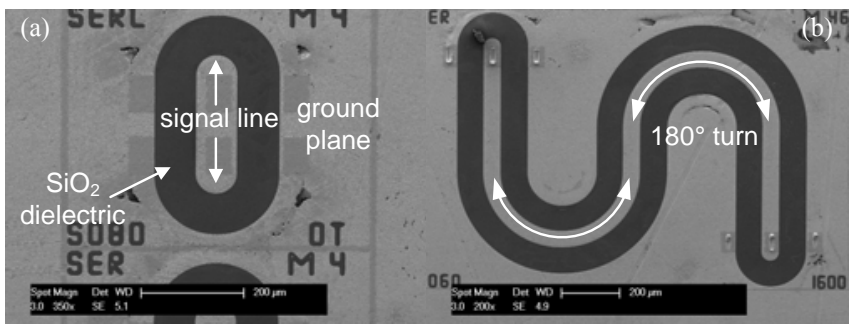

Fig. 4. SEM micrographs of SERC showing (a) a thru line and (b) a serpentine line with two $180^{\circ}$ turns.

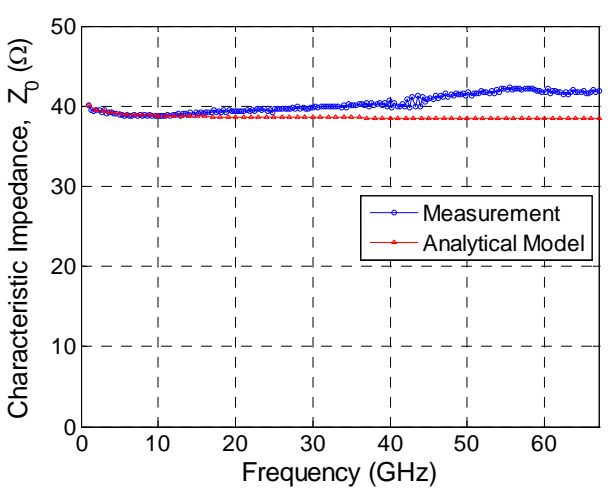

Fig. 5. Measurements/calculations of impedance vs. frequency.

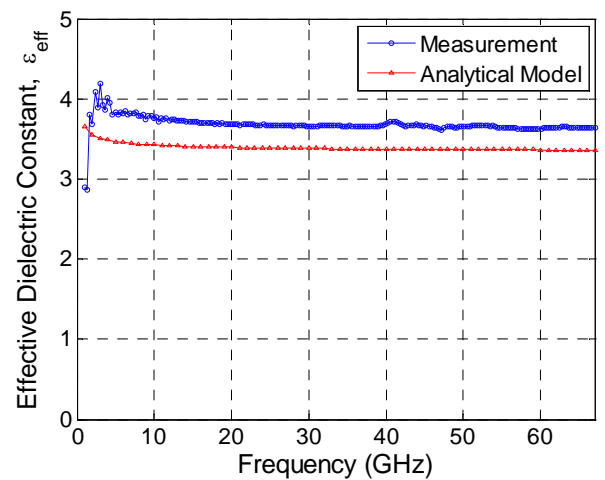

Fig. 6. Measurements/calculations of effective dielectric constant vs. frequency.

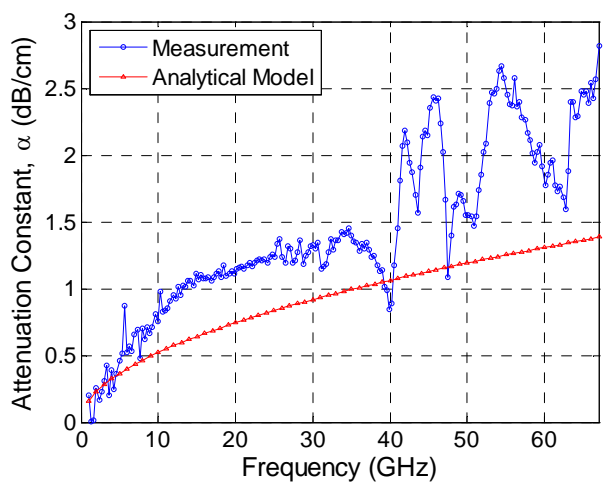

Fig. 7. Measurements/calculations of attenuation vs. frequency.

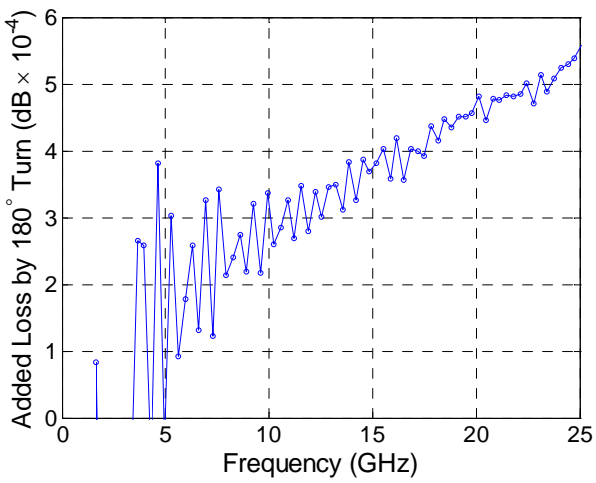

Fig. 8. Measurements of added loss by one $180^{\circ}$ turn. 